\title{
Acquired Phrenic Nerve Palsy
}

National Cancer Institute

\section{Source}

National Cancer Institute. Acquired Phrenic Nerve Palsy. NCI Thesaurus. Code C103189.

Damage to the phrenic nerve that results in paralysis of the hemidiaphragm and was not present at birth. 